Supplement of The Cryosphere, 14, 4279-4297, 2020

https://doi.org/10.5194/tc-14-4279-2020-supplement

(C) Author(s) 2020. This work is distributed under

the Creative Commons Attribution 4.0 License.

(c) (1)

Supplement of

\title{
The catastrophic thermokarst lake drainage events of 2018 in northwestern Alaska: fast-forward into the future
}

Ingmar Nitze et al.

Correspondence to: Ingmar Nitze (ingmar.nitze@awi.de)

The copyright of individual parts of the supplement might differ from the CC BY 4.0 License. 


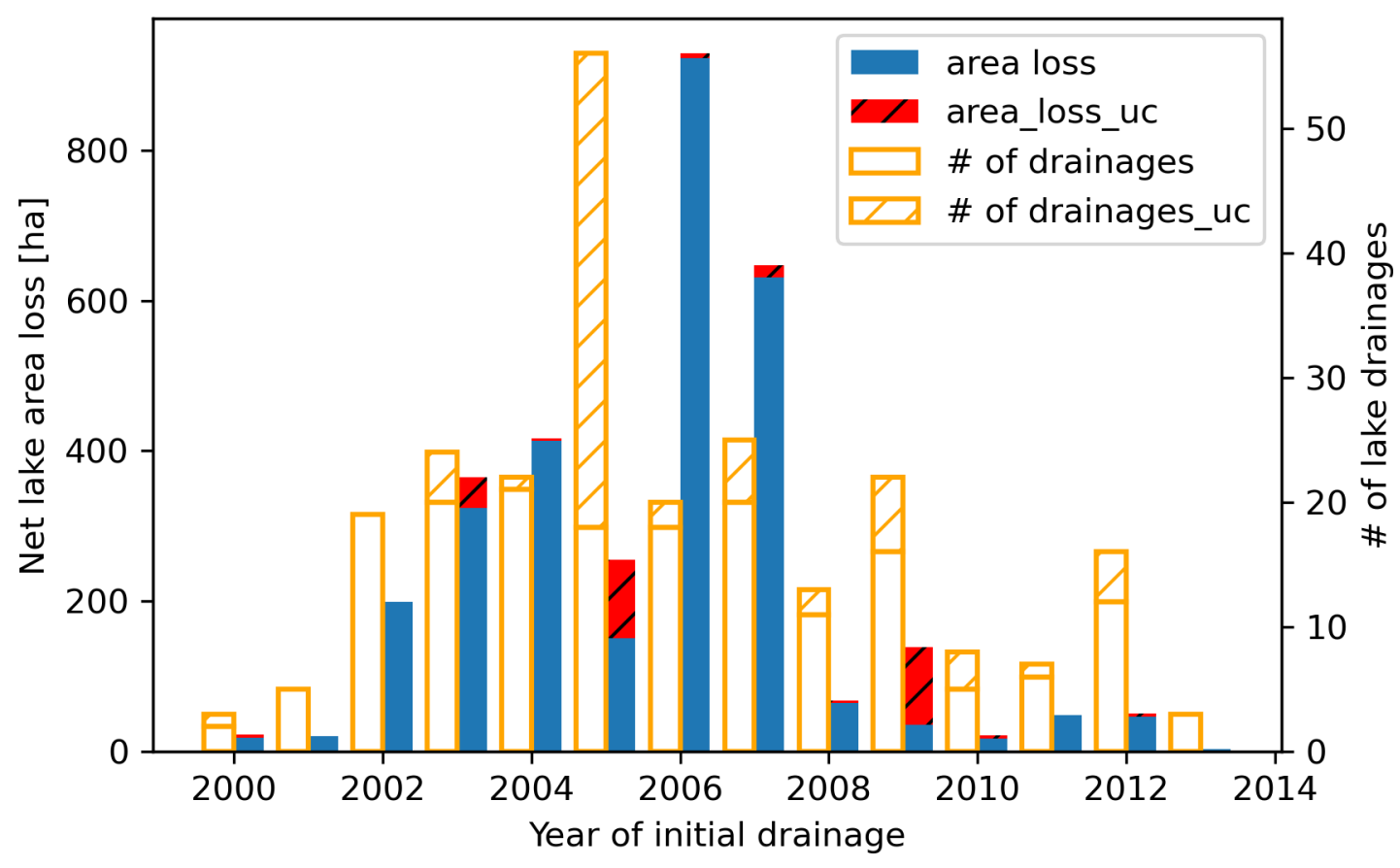

Figure S1: Identified drained lakes and area between 2000 and 2014. Uncertainties of number of lakes and drained lake area due to missing data or cloud cover in preceding years indicated.

Nov-Apr 2017-2018 L-OTI( $\left.{ }^{\circ} \mathrm{C}\right)$ Anomaly vs 1981-2010 0.46

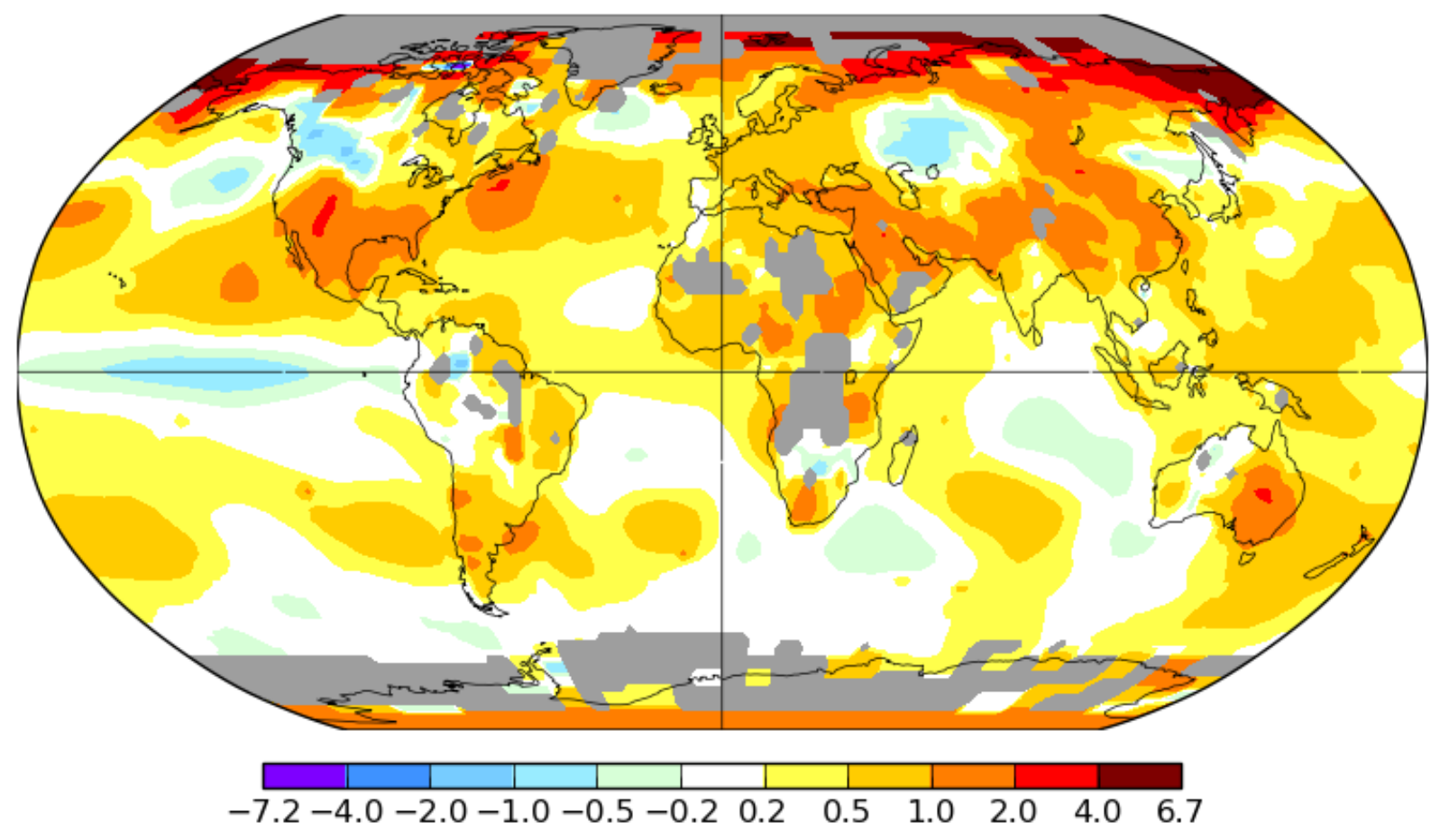


Figure S2: GHCNv4 re-analysis temperature anomaly November 2017 through April 2018 (GISTEMP Team, 2019).
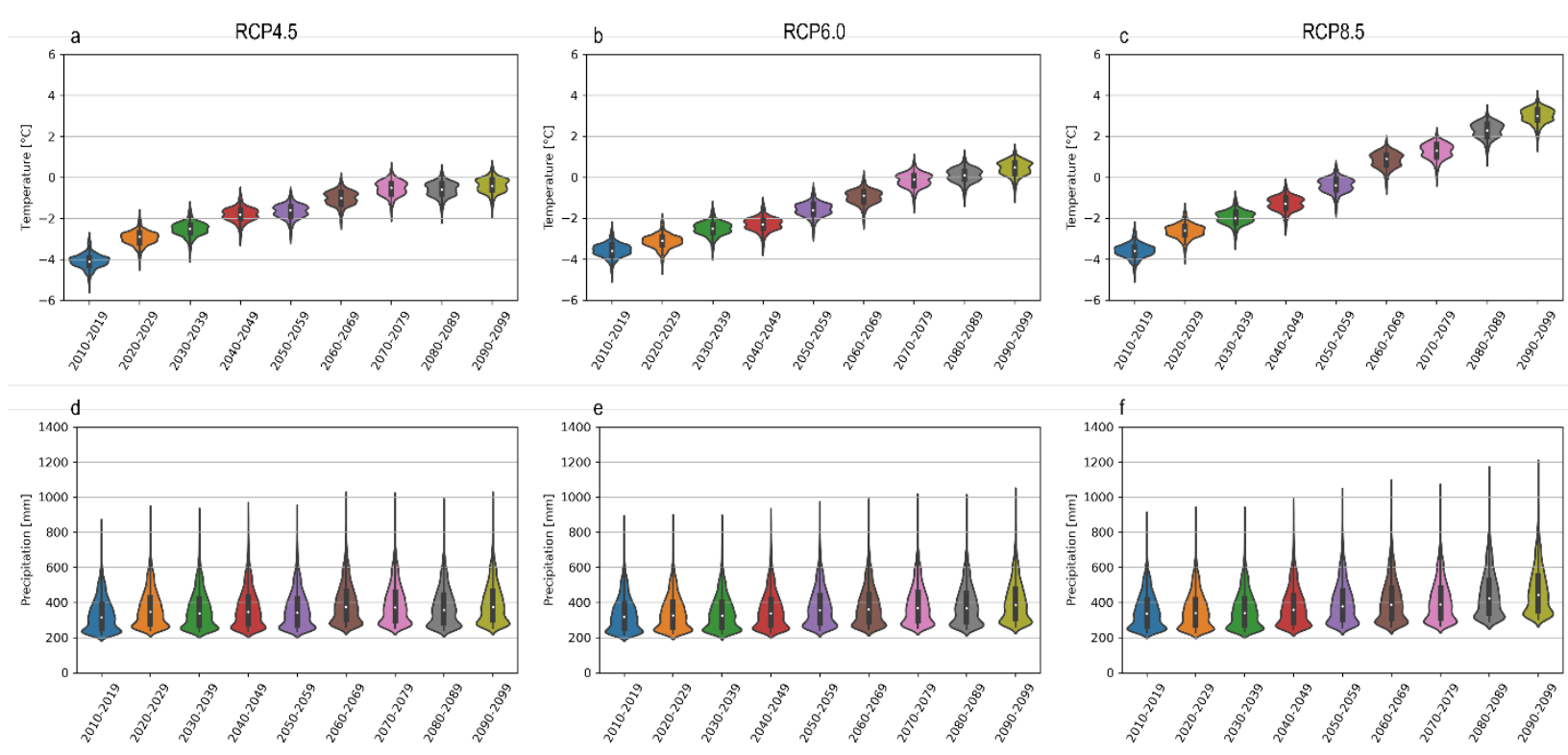

Figure S3: Decadal Regional SNAP Ensemble Climate Model Projections of the study region (2 km CMIP/AR5) of Scenarios RCP4.5 (a, d) , RCP6.0 (b, e), and RCP8.5 (c, f) for Temperatures (a-c) and Precipitation (d-f).

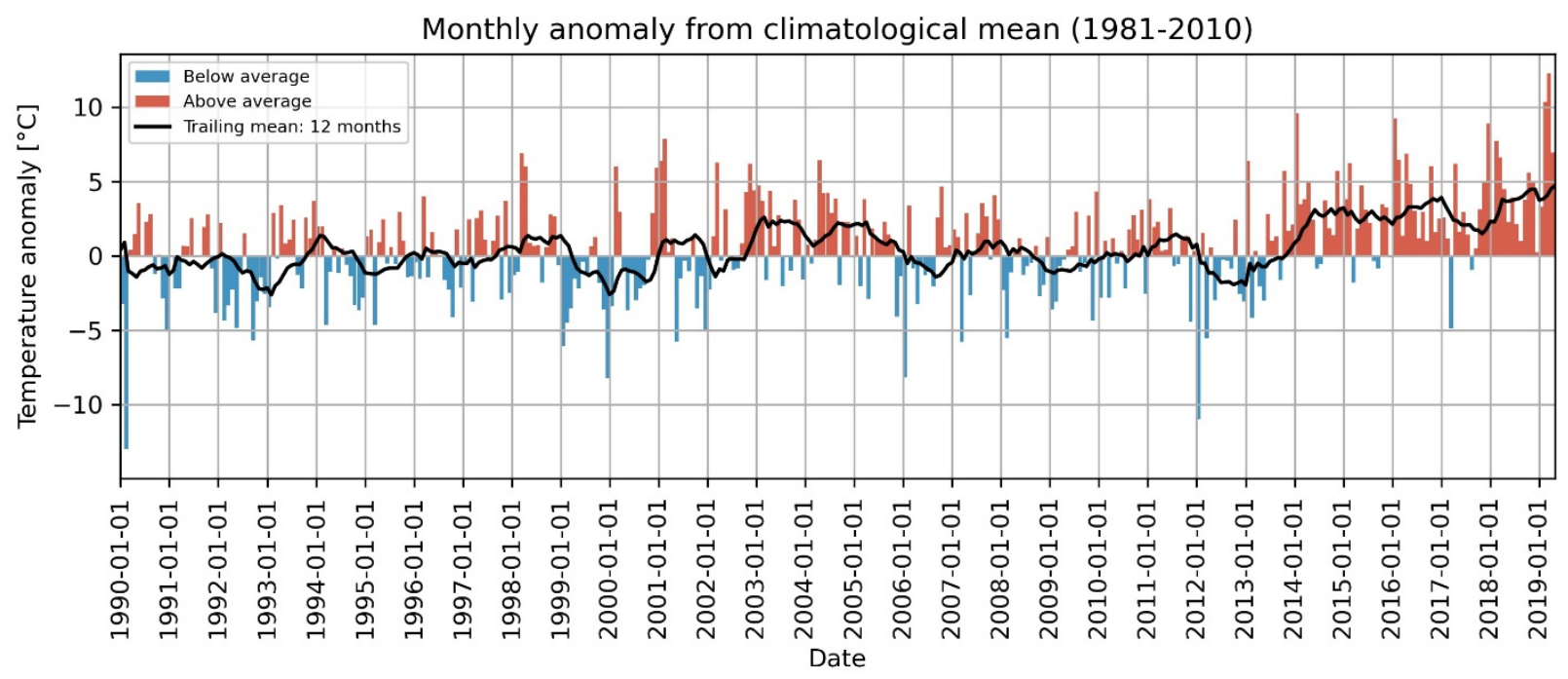

Figure S4: Anomaly of monthly temperatures from climatic mean (1981-2010) with 12 month trailing mean at weather station Kotzebue from 1990 through April 2019*. *Temperature measurements were not available from 1 May through 3 September 2019. 
Monthly anomaly from climatological mean (1981-2010)

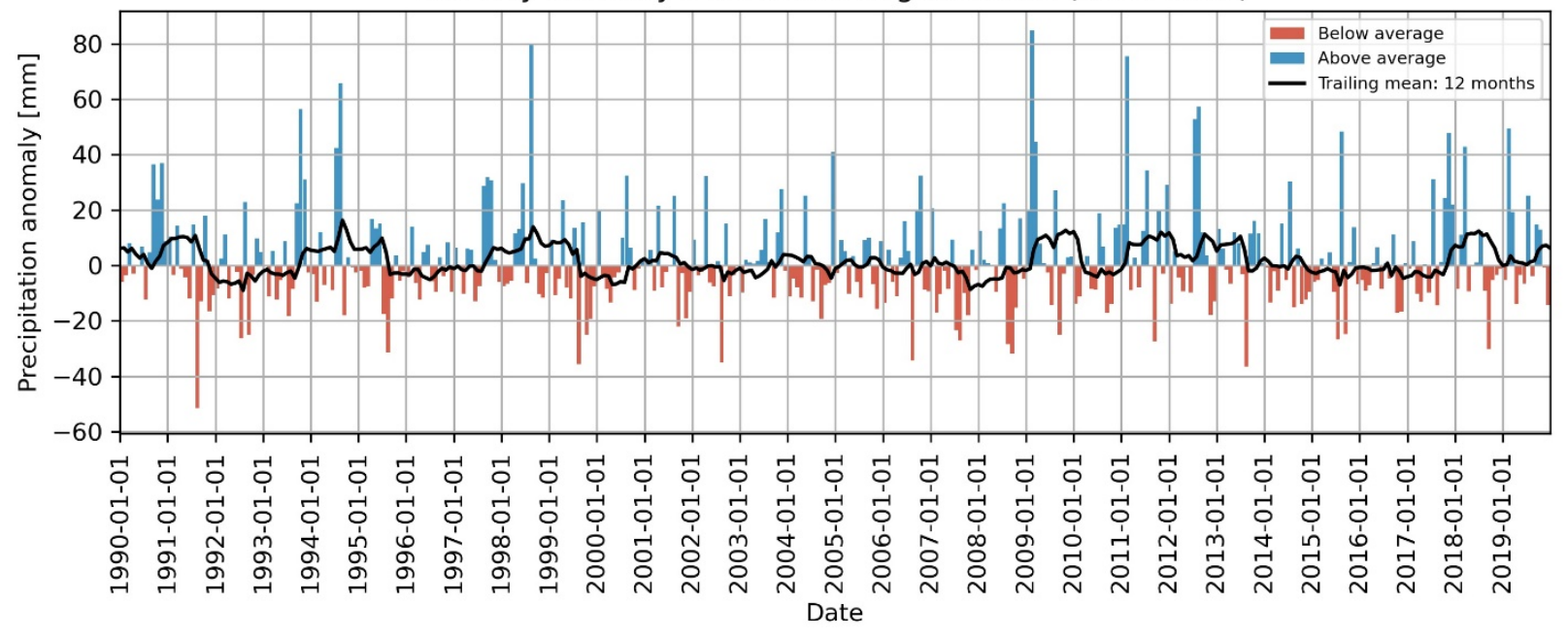

Figure S5: Anomaly of monthly precipitation from climatic mean (1981-2020) with 12 month trailing mean at weather station Kotzebue from 1990 through 2019. 
Table S1: Full List of Drained Lakes 2018 with Size rank of all lakes, LakeID, net change area from 2017 in ha and \% as well as area in 2017 and 2018, respectively. Table sorted by net area loss from 2017 to 2018. 


\begin{tabular}{|c|c|c|c|c|c|c|}
\hline & $\begin{array}{c}\text { Lake area rank } \\
2017 \\
\end{array}$ & LakeID & $\begin{array}{c}\text { Net change 2017-2018 } \\
\text { [ha] }\end{array}$ & $\begin{array}{c}\text { Net change 2017-2018 } \\
{[\%]}\end{array}$ & $\begin{array}{c}\text { Area } 2017 \\
\text { [ha] }\end{array}$ & Area 2018 [ha] \\
\hline 1 & 12 & 99368 & -332.04 & -91.24 & 363.92 & 31.88 \\
\hline 2 & 6 & 69152 & -258.8 & -34.307 & 754.36 & 495.56 \\
\hline 3 & 32 & 99230 & -185.12 & -99.698 & 185.68 & 0.56 \\
\hline 4 & 39 & 64656 & -164.6 & -99.83 & 164.88 & 0.28 \\
\hline 5 & 51 & 99492 & -132.12 & -100 & 132.12 & 0 \\
\hline 6 & 205 & 100218 & -28.48 & -78.242 & 36.4 & 7.92 \\
\hline 7 & 105 & 101659 & -27.56 & -41.531 & 66.36 & 38.8 \\
\hline 8 & 269 & 99545 & -26.12 & -97.317 & 26.84 & 0.72 \\
\hline 9 & 281 & 102499 & -25.72 & -100 & 25.72 & 0 \\
\hline 10 & 305 & 100470 & -23.2 & -100 & 23.2 & 0 \\
\hline 11 & 362 & 99557 & -19.48 & -100 & 19.48 & 0 \\
\hline 12 & 373 & 99550 & -18.84 & -98.95 & 19.04 & 0.2 \\
\hline 13 & 395 & 69287 & -18.24 & -100 & 18.24 & 0 \\
\hline 14 & 287 & 99756 & -17.24 & -68.413 & 25.2 & 7.96 \\
\hline 15 & 353 & 99505 & -12.16 & -60.558 & 20.08 & 7.92 \\
\hline 16 & 466 & 64659 & -11.8 & -76.425 & 15.44 & 3.64 \\
\hline 17 & 591 & 99465 & -11.64 & -97.651 & 11.92 & 0.28 \\
\hline 18 & 617 & 101224 & -11.36 & -100 & 11.36 & 0 \\
\hline 19 & 433 & 68965 & -9.4 & -57.457 & 16.36 & 6.96 \\
\hline 20 & 225 & 69482 & -8.64 & -26.024 & 33.2 & 24.56 \\
\hline 21 & 334 & 99205 & -7.68 & -35.622 & 21.56 & 13.88 \\
\hline 22 & 665 & 101680 & -7.64 & -73.745 & 10.36 & 2.72 \\
\hline 23 & 722 & 101909 & -7.56 & -81.116 & 9.32 & 1.76 \\
\hline 24 & 893 & 99516 & -7.4 & -100 & 7.4 & 0 \\
\hline 25 & 478 & 99257 & -7.28 & -47.895 & 15.2 & 7.92 \\
\hline 26 & 969 & 99519 & -6.68 & -100 & 6.68 & 0 \\
\hline 27 & 1047 & 100378 & -6.04 & $\mid-100$ & 6.04 & 0 \\
\hline 28 & 842 & 99108 & -5.76 & -73.469 & 7.84 & 2.08 \\
\hline 29 & 1139 & 69248 & -5.48 & -100 & 5.48 & 0 \\
\hline 30 & 361 & 100347 & -5.36 & -27.515 & 19.48 & 14.12 \\
\hline 31 & 1090 & 64520 & -5.2 & -89.655 & 5.8 & 0.6 \\
\hline 32 & 913 & 68788 & -5.2 & -72.222 & 7.2 & 2 \\
\hline 33 & 989 & 102024 & -5.12 & -78.528 & 6.52 & 1.4 \\
\hline 34 & 374 & 101693 & -4.96 & -26.105 & 19 & 14.04 \\
\hline 35 & 1211 & 99506 & -4.4 & -86.614 & 5.08 & 0.68 \\
\hline 36 & 1179 & 69590 & -4.32 & -81.818 & 5.28 & 0.96 \\
\hline 37 & 1135 & 101453 & -3.96 & -71.739 & 5.52 & 1.56 \\
\hline 38 & 825 & 100234 & -3.92 & -48.515 & 8.08 & 4.16 \\
\hline 39 & 1463 & 100268 & -3.92 & -100 & 3.92 & 0 \\
\hline 40 & 1505 & 101887 & -3.76 & -100 & 3.76 & 0 \\
\hline 41 & 950 & 99883 & -3.44 & -50 & 6.88 & 3.44 \\
\hline 42 & 1444 & 99224 & -3.32 & -83 & 4 & 0.68 \\
\hline 43 & 671 & 99179 & -3.28 & -32.031 & 10.24 & 6.96 \\
\hline 44 & 1666 & 101125 & -3.2 & -100 & 3.2 & 0 \\
\hline 45 & 1734 & 99421 & -3.04 & -100 & 3.04 & 0 \\
\hline 46 & 1700 & 101591 & -3 & -96.154 & 3.12 & 0.12 \\
\hline
\end{tabular}




\begin{tabular}{|c|c|c|c|c|c|c|}
\hline 47 & 737 & 69589 & -2.92 & -32.301 & 9.04 & 6.12 \\
\hline 48 & 892 & 71080 & -2.88 & -38.71 & 7.44 & 4.56 \\
\hline 49 & 1785 & 70973 & -2.88 & -100 & 2.88 & 0 \\
\hline 50 & 1667 & 70014 & -2.84 & -88.75 & 3.2 & 0.36 \\
\hline 51 & 1668 & 101752 & -2.48 & $\mid-77.5$ & 3.2 & 0.72 \\
\hline 52 & 912 & 63924 & -2.48 & -34.444 & 7.2 & 4.72 \\
\hline 53 & 986 & 69414 & -2.44 & -37.195 & 6.56 & 4.12 \\
\hline 54 & 2005 & 100177 & -2.44 & -100 & 2.44 & 0 \\
\hline 55 & 2033 & 71258 & -2.4 & -100 & 2.4 & 0 \\
\hline 56 & 2049 & 99198 & -2.28 & -96.61 & 2.36 & 0.08 \\
\hline 57 & 864 & 99403 & -2.28 & -29.687 & 7.68 & 5.4 \\
\hline 58 & 845 & 100528 & -2.28 & -29.082 & 7.84 & 5.56 \\
\hline 59 & 2058 & 99376 & -2.28 & -98.276 & 2.32 & 0.04 \\
\hline 60 & 1130 & 101246 & -2.2 & -39.568 & 5.56 & 3.36 \\
\hline 61 & 925 & 99568 & -2.2 & -31.25 & 7.04 & 4.84 \\
\hline 62 & 1374 & 99509 & -2.16 & -50.467 & 4.28 & 2.12 \\
\hline 63 & 1278 & 101618 & -2.08 & -43.697 & 4.76 & 2.68 \\
\hline 64 & 1964 & 70354 & -1.96 & -79.032 & 2.48 & 0.52 \\
\hline 65 & 1429 & 99583 & -1.92 & -47.525 & 4.04 & 2.12 \\
\hline 66 & 1851 & 101672 & -1.88 & -69.118 & 2.72 & 0.84 \\
\hline 67 & 1206 & 100034 & -1.84 & -35.938 & 5.12 & 3.28 \\
\hline 68 & 1386 & 100593 & -1.84 & -43.81 & 4.2 & 2.36 \\
\hline 69 & 1969 & 101135 & -1.76 & -70.968 & 2.48 & 0.72 \\
\hline 70 & 1832 & 69550 & -1.72 & -62.319 & 2.76 & 1.04 \\
\hline 71 & 991 & 69553 & -1.72 & -26.38 & 6.52 & 4.8 \\
\hline 72 & 1157 & 69526 & -1.68 & -31.111 & 5.4 & 3.72 \\
\hline 73 & 2143 & 102480 & -1.68 & -77.778 & 2.16 & 0.48 \\
\hline 74 & 2249 & 100562 & -1.64 & -83.673 & 1.96 & 0.32 \\
\hline 75 & 1905 & 100519 & -1.56 & -60 & 2.6 & 1.04 \\
\hline 76 & 1437 & 99153 & -1.52 & -38 & 4 & 2.48 \\
\hline 77 & 1379 & 69567 & -1.52 & -35.849 & 4.24 & 2.72 \\
\hline 78 & 1991 & 99200 & -1.48 & -60.656 & 2.44 & 0.96 \\
\hline 79 & 1150 & 99928 & -1.44 & -26.471 & 5.44 & 4 \\
\hline 80 & 2480 & 101396 & -1.4 & -83.333 & 1.68 & 0.28 \\
\hline 81 & 1664 & 100588 & -1.4 & -43.75 & 3.2 & 1.8 \\
\hline 82 & 1882 & 102605 & -1.36 & -50.746 & 2.68 & 1.32 \\
\hline 83 & 2521 & 99381 & -1.36 & -85 & 1.6 & 0.24 \\
\hline 84 & 1204 & 102098 & -1.32 & -25.781 & 5.12 & 3.8 \\
\hline 85 & 1684 & 100886 & -1.28 & -40.506 & 3.16 & 1.88 \\
\hline 86 & 2819 & 69037 & -1.28 & -100 & 1.28 & 0 \\
\hline 87 & 1507 & 99055 & \begin{tabular}{|l|}
-1.24 \\
\end{tabular} & \begin{tabular}{|l|}
-32.979 \\
\end{tabular} & 3.76 & 2.52 \\
\hline 88 & 2662 & 100644 & -1.24 & -83.784 & 1.48 & 0.24 \\
\hline 89 & 1820 & 99434 & -1.2 & -42.857 & 2.8 & 1.6 \\
\hline 90 & 1670 & 69677 & -1.16 & -36.25 & 3.2 & 2.04 \\
\hline 91 & 2917 & 99429 & -1.16 & -96.667 & 1.2 & 0.04 \\
\hline 92 & 1420 & 72236 & -1.16 & -28.431 & 4.08 & 2.92 \\
\hline 93 & 2791 & 99380 & -1.12 & -84.848 & 1.32 & 0.2 \\
\hline
\end{tabular}




\begin{tabular}{|c|c|c|c|c|c|c|}
\hline 94 & 1375 & 99744 & -1.12 & -26.168 & 4.28 & 3.16 \\
\hline 95 & 1709 & 100036 & -1.08 & -35.065 & 3.08 & 2 \\
\hline 96 & 1607 & 69938 & -1.08 & -31.765 & 3.4 & 2.32 \\
\hline 97 & 2266 & 99473 & -1.04 & -53.061 & 1.96 & 0.92 \\
\hline 98 & 2906 & 69649 & -1.04 & -86.667 & 1.2 & 0.16 \\
\hline 99 & 1486 & 102248 & -1 & -26.316 & 3.8 & 2.8 \\
\hline 100 & 1499 & 101422 & -1 & -26.596 & 3.76 & 2.76 \\
\hline 101 & 1671 & 69301 & -0.96 & -30 & 3.2 & 2.24 \\
\hline 102 & 1497 & 99418 & -0.96 & -25.532 & 3.76 & 2.8 \\
\hline 103 & 1566 & 99209 & -0.96 & -26.966 & 3.56 & 2.6 \\
\hline 104 & 2928 & 99571 & -0.92 & -76.667 & 1.2 & 0.28 \\
\hline 105 & 2474 & 72420 & -0.92 & -54.762 & 1.68 & 0.76 \\
\hline 106 & 2629 & 99208 & -0.92 & -62.162 & 1.48 & 0.56 \\
\hline 107 & 1773 & 68937 & -0.92 & -31.507 & 2.92 & 2 \\
\hline 108 & 1970 & 102593 & -0.88 & -35.484 & 2.48 & 1.6 \\
\hline 109 & 1743 & 99147 & -0.88 & -29.333 & 3 & 2.12 \\
\hline 110 & 1901 & 99235 & -0.84 & -32.308 & 2.6 & 1.76 \\
\hline 111 & 2154 & 101165 & -0.84 & -39.623 & 2.12 & 1.28 \\
\hline 112 & 2361 & 69614 & -0.84 & -45.652 & 1.84 & 1 \\
\hline 113 & 1728 & 100105 & -0.8 & -26.316 & 3.04 & 2.24 \\
\hline 114 & 2924 & 64366 & -0.8 & -66.667 & 1.2 & 0.4 \\
\hline 115 & 2074 & 99032 & -0.8 & -35.088 & 2.28 & 1.48 \\
\hline 116 & 2893 & 102777 & \begin{tabular}{|l|}
-0.8 \\
\end{tabular} & -66.667 & 1.2 & 0.4 \\
\hline 117 & 2879 & 99127 & -0.76 & -61.29 & 1.24 & 0.48 \\
\hline 118 & 1816 & 65563 & -0.76 & -27.143 & 2.8 & 2.04 \\
\hline 119 & 1749 & 100231 & -0.76 & -25.333 & 3 & 2.24 \\
\hline 120 & 2625 & 101086 & -0.76 & -51.351 & 1.48 & 0.72 \\
\hline 121 & 2362 & 70331 & -0.76 & -41.304 & 1.84 & 1.08 \\
\hline 122 & 2518 & 101460 & -0.76 & -47.5 & 1.6 & 0.84 \\
\hline 123 & 1845 & 69634 & -0.76 & -27.941 & 2.72 & 1.96 \\
\hline 124 & 1811 & 99389 & -0.72 & -25.714 & 2.8 & 2.08 \\
\hline 125 & 2071 & 99058 & -0.72 & -31.579 & 2.28 & 1.56 \\
\hline 126 & 2231 & 69407 & \begin{tabular}{|l|}
-0.72 \\
\end{tabular} & -36 & 2 & 1.28 \\
\hline 127 & 2162 & 101614 & \begin{tabular}{|l|}
-0.68 \\
\end{tabular} & -32.075 & 2.12 & 1.44 \\
\hline 128 & 2524 & 99145 & -0.68 & -42.5 & 1.6 & 0.92 \\
\hline 129 & 2270 & 101234 & -0.68 & -34.694 & 1.96 & 1.28 \\
\hline 130 & 2657 & 69609 & -0.68 & -45.946 & 1.48 & 0.8 \\
\hline 131 & 2892 & 71776 & -0.68 & -56.667 & 1.2 & 0.52 \\
\hline 132 & 2015 & 68768 & -0.68 & -28.333 & 2.4 & 1.72 \\
\hline 133 & 2867 & 71246 & \begin{tabular}{|l|}
-0.64 \\
\end{tabular} & -51.613 & 1.24 & 0.6 \\
\hline 134 & 3129 & 99104 & \begin{tabular}{|l|}
-0.64 \\
\end{tabular} & -64 & 1 & 0.36 \\
\hline 135 & 2467 & 101650 & -0.64 & -38.095 & 1.68 & 1.04 \\
\hline 136 & 2011 & 100913 & -0.64 & -26.667 & 2.4 & 1.76 \\
\hline 137 & 2650 & 99367 & -0.6 & -40.541 & 1.48 & 0.88 \\
\hline 138 & 2175 & 100645 & -0.6 & -28.846 & 2.08 & 1.48 \\
\hline 139 & 2235 & 99532 & -0.6 & -30 & 2 & 1.4 \\
\hline 140 & 2324 & 100157 & -0.6 & -31.915 & 1.88 & 1.28 \\
\hline
\end{tabular}




\begin{tabular}{|c|c|c|c|c|c|c|}
\hline 141 & 2647 & 72042 & -0.6 & -40.541 & 1.48 & 0.88 \\
\hline 142 & 2240 & 101652 & -0.56 & -28 & 2 & 1.44 \\
\hline 143 & 2197 & 100327 & -0.56 & -27.451 & 2.04 & 1.48 \\
\hline 144 & 2913 & 99363 & \begin{tabular}{|l|}
-0.56 \\
\end{tabular} & -46.667 & 1.2 & 0.64 \\
\hline 145 & 2441 & 69803 & -0.56 & -32.558 & 1.72 & 1.16 \\
\hline 146 & 2593 & 99062 & -0.56 & -36.842 & 1.52 & 0.96 \\
\hline 147 & 2322 & 102732 & -0.56 & \begin{tabular}{|l|}
-29.787 \\
\end{tabular} & 1.88 & 1.32 \\
\hline 148 & 2400 & 99146 & -0.56 & -31.818 & 1.76 & 1.2 \\
\hline 149 & 2886 & 99800 & \begin{tabular}{|l|}
-0.52 \\
\end{tabular} & -41.935 & 1.24 & 0.72 \\
\hline 150 & 2911 & 99668 & -0.52 & -43.333 & 1.2 & 0.68 \\
\hline 151 & 2957 & 100705 & -0.52 & -44.828 & 1.16 & 0.64 \\
\hline 152 & 2719 & 99374 & -0.52 & -37.143 & 1.4 & 0.88 \\
\hline 153 & 3148 & 101915 & -0.48 & -48 & 1 & 0.52 \\
\hline 154 & 3084 & 100836 & -0.48 & -46.154 & 1.04 & 0.56 \\
\hline 155 & 2552 & 72962 & -0.48 & -30.769 & 1.56 & 1.08 \\
\hline 156 & 2705 & 101210 & \begin{tabular}{|l|}
-0.48 \\
\end{tabular} & -34.286 & 1.4 & 0.92 \\
\hline 157 & 2652 & 71238 & \begin{tabular}{|l|}
-0.48 \\
\end{tabular} & -32.432 & 1.48 & 1 \\
\hline 158 & 2706 & 73001 & -0.48 & -34.286 & 1.4 & 0.92 \\
\hline 159 & 2416 & 99551 & \begin{tabular}{|l|}
-0.48 \\
\end{tabular} & -27.273 & 1.76 & 1.28 \\
\hline 160 & 2454 & 99214 & -0.48 & -27.907 & 1.72 & 1.24 \\
\hline 161 & 2804 & 71574 & -0.48 & -36.364 & 1.32 & 0.84 \\
\hline 162 & 2309 & 100926 & \begin{tabular}{|l|}
-0.48 \\
\end{tabular} & -25.532 & 1.88 & 1.4 \\
\hline 163 & 2499 & 102724 & \begin{tabular}{|l|}
-0.48 \\
\end{tabular} & -29.268 & 1.64 & 1.16 \\
\hline 164 & 2679 & 101555 & \begin{tabular}{|l|}
-0.48 \\
\end{tabular} & -33.333 & 1.44 & 0.96 \\
\hline 165 & 2585 & 100619 & -0.44 & $\mid-28.947$ & 1.52 & 1.08 \\
\hline 166 & 2721 & 101530 & \begin{tabular}{|l|}
-0.44 \\
\end{tabular} & -31.429 & 1.4 & 0.96 \\
\hline 167 & 2813 & 71516 & -0.44 & -33.333 & 1.32 & 0.88 \\
\hline 168 & 2856 & 72309 & -0.44 & -35.484 & 1.24 & 0.8 \\
\hline 169 & 3034 & 102279 & -0.44 & -40.741 & 1.08 & 0.64 \\
\hline 170 & 2634 & 99143 & -0.44 & -29.73 & 1.48 & 1.04 \\
\hline 171 & 2735 & 69040 & -0.44 & -31.429 & 1.4 & 0.96 \\
\hline 172 & 2643 & 99355 & -0.4 & -27.027 & 1.48 & 1.08 \\
\hline 173 & 2693 & 100512 & -0.4 & -27.778 & 1.44 & 1.04 \\
\hline 174 & 2680 & 71213 & \begin{tabular}{|l|}
-0.4 \\
\end{tabular} & -27.778 & 1.44 & 1.04 \\
\hline 175 & 2687 & 71095 & -0.4 & -27.778 & 1.44 & 1.04 \\
\hline 176 & 2846 & 99082 & -0.4 & -31.25 & 1.28 & 0.88 \\
\hline 177 & 2723 & 71207 & -0.4 & -28.571 & 1.4 & 1 \\
\hline 178 & 2824 & 71673 & -0.4 & -31.25 & 1.28 & 0.88 \\
\hline 179 & 2743 & 99266 & \begin{tabular}{|l|}
-0.4 \\
\end{tabular} & -29.412 & 1.36 & 0.96 \\
\hline 180 & 2798 & 100184 & -0.4 & -30.303 & 1.32 & 0.92 \\
\hline 181 & 2646 & 100838 & -0.4 & -27.027 & 1.48 & 1.08 \\
\hline 182 & 2836 & 70968 & -0.4 & -31.25 & 1.28 & 0.88 \\
\hline 183 & 2591 & 64067 & -0.4 & -26.316 & 1.52 & 1.12 \\
\hline 184 & 3010 & 99503 & -0.4 & -35.714 & 1.12 & 0.72 \\
\hline 185 & 2968 & 99162 & -0.36 & -31.034 & 1.16 & 0.8 \\
\hline 186 & 2925 & 100752 & -0.36 & -30 & 1.2 & 0.84 \\
\hline 187 & 2964 & 70333 & \begin{tabular}{|l|}
-0.36 \\
\end{tabular} & -31.034 & 1.16 & 0.8 \\
\hline
\end{tabular}




\begin{tabular}{|l|l|l|l|l|l|l|}
\hline 188 & 2771 & 101998 & -0.36 & -27.273 & 1.32 & 0.96 \\
\hline 189 & 2810 & 68981 & -0.36 & -27.273 & 1.32 & 0.96 \\
\hline 190 & 3081 & 101908 & -0.32 & -30.769 & 1.04 & 0.72 \\
\hline 191 & 3105 & 100114 & -0.32 & -32 & 1 & 0.68 \\
\hline 192 & 2939 & 99797 & -0.32 & -27.586 & 1.16 & 0.84 \\
\hline 193 & 3024 & 69362 & -0.32 & -29.63 & 1.08 & 0.76 \\
\hline 194 & 3097 & 99362 & -0.28 & -26.923 & 1.04 & 0.76 \\
\hline 195 & 3042 & 99652 & -0.28 & -25.926 & 1.08 & 0.8 \\
\hline
\end{tabular}

Table S2: Full List of Drained Lakes (>25\% loss, >1ha size) 1999-2014 with Size rank of all lakes, LakeID, net change area from 1999to 2014 in ha and \% as well as lake area in 1999 and 2014, respectively. Table sorted by net area loss from 1999 to 2014. Year of drainage for lakes without "Year Drained" value could not be retrieved due to insufficient temporal or spatial resolution.

\begin{tabular}{|c|c|c|c|c|c|c|}
\hline & LakeID & $\begin{array}{c}\text { Net change 1999- } \\
2014 \text { [ha] }\end{array}$ & \begin{tabular}{|c|} 
Net change \\
1999-2014 [\%]
\end{tabular} & Area 1999 [ha] & Area 2014 [ha] & Year Drained \\
\hline 1 & 101282 & -568.92 & -97.95 & 580.8 & 11.88 & 2007 \\
\hline 2 & 99433 & -373.29 & -99.63 & 374.67 & 1.37 & 2006 \\
\hline 3 & 99313 & -299.98 & -78.77 & 380.84 & 80.85 & 2006 \\
\hline 4 & 100588 & -208.53 & -94.69 & 220.22 & 11.69 & 2004 \\
\hline 5 & 99624 & -113.43 & -99.55 & 113.94 & 0.52 & 2006 \\
\hline 6 & 101659 & -79.32 & -31.55 & 251.42 & 172.1 & 2009 \\
\hline 7 & 99505 & -76.16 & -62.7 & 121.48 & 45.31 & 2003 \\
\hline 8 & 100505 & -74.27 & -28.86 & 257.36 & 183.08 & 2003 \\
\hline 9 & 101402 & -65.62 & -98.3 & 66.75 & 1.14 & 2003 \\
\hline 10 & 101844 & -56.5 & -99.06 & 57.03 & 0.54 & 2004 \\
\hline 11 & 101223 & -45.54 & -73.06 & 62.34 & 16.79 & 2002 \\
\hline 12 & 100387 & -41.26 & -64.37 & 64.1 & 22.84 & 2006 \\
\hline 13 & 101624 & -40.72 & -95.74 & 42.54 & 1.81 & 2002 \\
\hline 14 & 100506 & -40.55 & -37.3 & 108.69 & 68.15 & 2005 \\
\hline 15 & 101888 & -38.56 & -63.33 & 60.88 & 22.33 & 2003 \\
\hline 16 & 102655 & -34.86 & -86.03 & 40.53 & 5.66 & 2006 \\
\hline 17 & 102007 & -34.32 & -69.57 & 49.33 & 15.01 & 2003 \\
\hline 18 & 102196 & -32.29 & -43.42 & 74.37 & 42.08 & 2004 \\
\hline 19 & 69745 & -28.41 & -96.54 & 29.43 & 1.02 & 2002 \\
\hline 20 & 101228 & -27.6 & -92.14 & 29.96 & 2.36 & 2002 \\
\hline 21 & 100573 & -26.71 & -97.67 & 27.35 & 0.64 & 2004 \\
\hline 22 & 102395 & -26.69 & -36.38 & 73.36 & 46.67 & 2012 \\
\hline 23 & 102593 & -25.91 & -82.18 & 31.53 & 5.62 & 2003 \\
\hline 24 & 102399 & -24.18 & -98.35 & 24.58 & 0.4 & 2002 \\
\hline 25 & 99484 & -22.78 & -31.53 & 72.26 & 49.47 & 2005 \\
\hline 26 & 101752 & -19.94 & -53.56 & 37.23 & 17.29 & 2009 \\
\hline 27 & 100031 & -18.33 & -77.19 & 23.75 & 5.42 & -- \\
\hline 28 & 101374 & -16.45 & -31.54 & 52.14 & 35.7 & 2005 \\
\hline 29 & 102098 & -16.25 & -57.56 & 28.23 & 11.98 & 2011 \\
\hline 30 & 102803 & -15.77 & -98.05 & 16.09 & 0.31 & 2000 \\
\hline 31 & 99896 & -15.28 & -93.46 & 16.35 & 1.07 & 2005 \\
\hline 32 & 102476 & -14.94 & -47.36 & 31.55 & 16.61 & 2011 \\
\hline
\end{tabular}




\begin{tabular}{|c|c|c|c|c|c|c|}
\hline 33 & 99914 & -14.79 & \begin{tabular}{|l|}
-99.09 \\
\end{tabular} & 14.92 & 0.14 & 2005 \\
\hline 34 & 102358 & -14.6 & -87.77 & 16.63 & 2.03 & 2004 \\
\hline 35 & 102775 & -13.7 & -96 & 14.27 & 0.57 & 2008 \\
\hline 36 & 102759 & -13.22 & -99.58 & 13.28 & 0.06 & 2008 \\
\hline 37 & 102284 & -12.96 & -66.87 & 19.38 & 6.42 & 2004 \\
\hline 38 & 68981 & -12.06 & $\mid-86.72$ & 13.91 & 1.85 & 2007 \\
\hline 39 & 99588 & -12.02 & $\mid-97.95$ & 12.27 & 0.25 & 2006 \\
\hline 40 & 69026 & -10.69 & -99.58 & 10.74 & 0.05 & 2005 \\
\hline 41 & 101520 & -10.51 & -87.16 & 12.06 & 1.55 & 2006 \\
\hline 42 & 102767 & -10.27 & -99.03 & 10.37 & 0.1 & 2005 \\
\hline 43 & 99210 & -10.27 & -25.1 & 40.91 & 30.64 & 2001 \\
\hline 44 & 102335 & -9.83 & -42.34 & 23.21 & 13.38 & 2004 \\
\hline 45 & 102409 & \begin{tabular}{|l|} 
\\
\end{tabular} & -57.05 & 17.17 & 7.38 & 2004 \\
\hline 46 & 102491 & -9.19 & -99.36 & 9.24 & 0.06 & 2003 \\
\hline 47 & 99461 & -9.13 & -93.44 & 9.77 & 0.64 & 2005 \\
\hline 48 & 102454 & \begin{tabular}{|l|}
-9.09 \\
\end{tabular} & -54.88 & 16.57 & 7.48 & 2006 \\
\hline 49 & 69195 & \begin{tabular}{|l|}
-9.08 \\
\end{tabular} & -95.77 & 9.48 & 0.4 & 2008 \\
\hline 50 & 101809 & -8.6 & -99.64 & 8.63 & 0.03 & 2004 \\
\hline 51 & 99360 & -8.51 & -86.96 & 9.78 & 1.28 & 2008 \\
\hline 52 & 99617 & -8.39 & -52.15 & 16.09 & 7.7 & 2010 \\
\hline 53 & 71375 & -7.95 & -99.29 & 8 & 0.06 & 2007 \\
\hline 54 & 99369 & -7.79 & -71.78 & 10.86 & 3.06 & 2011 \\
\hline 55 & 102768 & -7.22 & -97.14 & 7.44 & 0.21 & 2005 \\
\hline \begin{tabular}{|l|}
56 \\
\end{tabular} & 99997 & -6.85 & -31.99 & 21.4 & 14.56 & \begin{tabular}{|l}
-- \\
\end{tabular} \\
\hline \begin{tabular}{|l|}
57 \\
\end{tabular} & 69571 & -6.83 & -61.4 & 11.12 & 4.29 & 2002 \\
\hline \begin{tabular}{|l|}
58 \\
\end{tabular} & 102300 & -6.46 & -99.6 & 6.48 & 0.03 & 2004 \\
\hline \begin{tabular}{|l|}
59 \\
\end{tabular} & 72895 & -6.27 & -84.12 & 7.45 & 1.18 & 2005 \\
\hline 60 & 100825 & -6.2 & -95.49 & 6.49 & 0.29 & 2005 \\
\hline 61 & 101272 & -6.07 & -66.75 & 9.09 & 3.02 & 2007 \\
\hline 62 & 101287 & -5.94 & -29.85 & 19.91 & 13.96 & 2003 \\
\hline 63 & 101470 & -5.91 & -74.07 & 7.98 & 2.07 & 2004 \\
\hline 64 & 100248 & \begin{tabular}{|l|}
-5.88 \\
\end{tabular} & -25.25 & 23.28 & 17.4 & 2011 \\
\hline 65 & 71194 & -5.63 & -66.56 & 8.45 & 2.83 & 2012 \\
\hline 66 & 99823 & -5.61 & -85.65 & 6.56 & 0.94 & \begin{tabular}{|l|}
--- \\
\end{tabular} \\
\hline 67 & 100790 & -5.59 & -28.75 & 19.44 & 13.85 & 2005 \\
\hline 68 & 102227 & -5.4 & -69.25 & 7.8 & 2.4 & 2004 \\
\hline 69 & 101964 & -5.11 & -77.59 & 6.59 & 1.48 & 2006 \\
\hline 70 & 101969 & -5.05 & -86.09 & 5.87 & 0.82 & 2007 \\
\hline \begin{tabular}{|l|}
71 \\
\end{tabular} & 102451 & -5.03 & -84.31 & 5.96 & 0.94 & 2004 \\
\hline 72 & 100450 & -4.78 & -58.97 & 8.11 & 3.33 & 2007 \\
\hline 73 & 101773 & -4.65 & -45.04 & 10.33 & 5.68 & 2007 \\
\hline \begin{tabular}{|l|}
74 \\
\end{tabular} & 102623 & -4.64 & -60.49 & 7.67 & 3.03 & 2008 \\
\hline 75 & 100551 & -4.62 & -83.15 & 5.56 & 0.94 & 2005 \\
\hline 76 & 102746 & -4.61 & -84.46 & 5.46 & 0.85 & 2009 \\
\hline 77 & 72334 & -4.54 & -96.87 & 4.68 & 0.15 & 2007 \\
\hline 78 & 100663 & -4.48 & -32.85 & 13.64 & 9.16 & 2002 \\
\hline 79 & 71290 & -4.47 & -96.71 & 4.63 & 0.15 & 2010 \\
\hline
\end{tabular}




\begin{tabular}{|c|c|c|c|c|c|c|}
\hline 80 & 64366 & -4.45 & -30.45 & 14.61 & 10.16 & 2009 \\
\hline 81 & 102729 & -4.42 & -25.52 & 17.34 & 12.91 & 2009 \\
\hline 82 & 102717 & -4.28 & -96.92 & 4.42 & 0.14 & 2006 \\
\hline 83 & 101961 & -4.22 & -39.48 & 10.68 & 6.46 & 2006 \\
\hline 84 & 102537 & -4.22 & -48.72 & 8.67 & 4.45 & 2000 \\
\hline 85 & 100737 & $\mid-4.15$ & -90.56 & 4.58 & 0.43 & 2005 \\
\hline 86 & 65014 & -4 & -70.71 & 5.66 & 1.66 & 2007 \\
\hline 87 & 102222 & -3.93 & \begin{tabular}{|l|}
-97.45 \\
\end{tabular} & 4.03 & 0.1 & 2005 \\
\hline 88 & 102523 & -3.91 & -74.02 & 5.28 & 1.37 & 2004 \\
\hline 89 & 72926 & -3.86 & -98.82 & 3.9 & 0.05 & 2005 \\
\hline 90 & 99804 & -3.76 & -35.27 & 10.66 & 6.9 & 2014 \\
\hline 91 & 100584 & -3.73 & -78.61 & 4.74 & 1.01 & 2006 \\
\hline 92 & 101178 & -3.56 & -33.45 & 10.66 & 7.09 & 2001 \\
\hline 93 & 100797 & -3.55 & -73.29 & 4.85 & 1.3 & 2003 \\
\hline 94 & 102494 & -3.52 & -93.16 & 3.78 & 0.26 & 2003 \\
\hline 95 & 102178 & \begin{tabular}{|l|}
-3.51 \\
\end{tabular} & \begin{tabular}{|l|}
-82.03 \\
\end{tabular} & 4.28 & 0.77 & 2007 \\
\hline 96 & 99850 & -3.5 & -95.97 & 3.65 & 0.15 & --- \\
\hline 97 & 102616 & \begin{tabular}{|l|}
-3.42 \\
\end{tabular} & -98.23 & 3.48 & 0.06 & 2007 \\
\hline 98 & 100861 & -3.41 & -41.5 & 8.21 & 4.8 & 2003 \\
\hline 99 & 69275 & \begin{tabular}{|l|} 
\\
\end{tabular} & -78.12 & 4.31 & 0.94 & 2009 \\
\hline 100 & 101313 & -3.33 & -71.22 & 4.68 & 1.35 & 2005 \\
\hline 101 & 99374 & -3.32 & \begin{tabular}{|l|}
-63.2 \\
\end{tabular} & 5.26 & 1.94 & 2008 \\
\hline 102 & 102773 & -3.31 & -92.18 & 3.59 & 0.28 & 2005 \\
\hline 103 & 100800 & -3.19 & -98.95 & 3.22 & 0.03 & 2005 \\
\hline 104 & 100988 & -3.18 & -86.93 & 3.66 & 0.48 & 2005 \\
\hline 105 & 102091 & -3.16 & -80.44 & 3.93 & 0.77 & 2001 \\
\hline 106 & 100261 & -3.13 & -84.93 & 3.68 & 0.55 & 2007 \\
\hline 107 & 102243 & -3.08 & -48.8 & 6.32 & 3.23 & 2005 \\
\hline 108 & 102440 & -3.08 & -80.58 & 3.82 & 0.74 & 2007 \\
\hline 109 & 100727 & -3.07 & -51.52 & 5.96 & 2.89 & 2002 \\
\hline 110 & 102612 & -3.06 & -95.21 & 3.21 & 0.15 & 2008 \\
\hline 111 & 101166 & -3.05 & -93.06 & 3.28 & 0.23 & 2002 \\
\hline 112 & 102149 & -3.05 & -99.46 & 3.07 & 0.02 & 2005 \\
\hline 113 & 102489 & -2.96 & -82.74 & 3.58 & 0.62 & 2003 \\
\hline 114 & 102670 & -2.95 & -94.28 & 3.13 & 0.18 & 2010 \\
\hline 115 & 69720 & -2.95 & -99.5 & 2.96 & 0.01 & 2005 \\
\hline 116 & 101072 & -2.92 & \begin{tabular}{|l|}
-96.03 \\
\end{tabular} & 3.04 & 0.12 & 2005 \\
\hline \begin{tabular}{|l|}
117 \\
\end{tabular} & 99329 & -2.9 & -98.61 & 2.94 & 0.04 & 2003 \\
\hline 118 & 69849 & -2.9 & -56.16 & 5.16 & 2.26 & 2008 \\
\hline \begin{tabular}{|l|}
119 \\
\end{tabular} & 102774 & -2.88 & -60.84 & 4.73 & 1.85 & 2006 \\
\hline 120 & 101184 & -2.83 & -96.5 & 2.94 & 0.1 & 2008 \\
\hline 121 & 101439 & -2.82 & -49.33 & 5.72 & 2.9 & 2008 \\
\hline 122 & 100827 & -2.81 & -35.25 & 7.97 & 5.16 & 2002 \\
\hline 123 & 101290 & -2.8 & -98.9 & 2.83 & 0.03 & 2003 \\
\hline 124 & 101967 & \begin{tabular}{|l|}
-2.79 \\
\end{tabular} & -57.45 & 4.85 & 2.07 & 2012 \\
\hline 125 & 102723 & -2.75 & -99.59 & 2.76 & 0.01 & 2006 \\
\hline 126 & 99732 & -2.73 & -97.56 & 2.8 & 0.07 & 2000 \\
\hline
\end{tabular}




\begin{tabular}{|c|c|c|c|c|c|c|}
\hline 127 & 69195 & -2.69 & -99.42 & 2.71 & 0.02 & --- \\
\hline 128 & 99181 & -2.65 & -99.41 & 2.67 & 0.02 & 2001 \\
\hline 129 & 99764 & -2.62 & $\mid-78.31$ & 3.35 & 0.73 & 2006 \\
\hline 130 & 69280 & -2.54 & -25.9 & 9.81 & 7.27 & --- \\
\hline 131 & 102304 & -2.53 & -97.74 & 2.59 & 0.06 & 2006 \\
\hline 132 & 71625 & -2.43 & -98.07 & 2.47 & 0.05 & 2005 \\
\hline 133 & 100417 & -2.43 & -63.55 & 3.83 & 1.4 & 2009 \\
\hline 134 & 102481 & -2.38 & -98.77 & 2.41 & 0.03 & 2004 \\
\hline 135 & 101233 & -2.36 & -76.85 & 3.07 & 0.71 & 2003 \\
\hline 136 & 101590 & -2.35 & -45.22 & 5.19 & 2.84 & 2002 \\
\hline 137 & 72808 & $\mid-2.3$ & -99.24 & 2.32 & 0.02 & 2005 \\
\hline 138 & 101191 & -2.24 & \begin{tabular}{|l|}
-74.7 \\
\end{tabular} & 2.99 & 0.76 & 2005 \\
\hline 139 & 102602 & -2.23 & -84.87 & 2.62 & 0.4 & 2004 \\
\hline 140 & 102547 & -2.23 & -31.23 & 7.15 & 4.92 & 2007 \\
\hline 141 & 99530 & -2.21 & -35.48 & 6.23 & 4.02 & 2012 \\
\hline 142 & 69271 & \begin{tabular}{|l|}
-2.18 \\
\end{tabular} & -96.15 & 2.27 & 0.09 & --- \\
\hline 143 & 102144 & -2.13 & -56.53 & 3.76 & 1.64 & 2010 \\
\hline 144 & 100932 & \begin{tabular}{|l|}
-2.1 \\
\end{tabular} & -98.28 & 2.14 & 0.04 & 2005 \\
\hline 145 & 101768 & -2.09 & -39.72 & 5.27 & 3.17 & 2003 \\
\hline 146 & 100972 & -2.08 & -53.67 & 3.88 & 1.8 & 2006 \\
\hline 147 & 69816 & -2.06 & -87.27 & 2.36 & 0.3 & 2008 \\
\hline 148 & 102674 & -2.05 & \begin{tabular}{|l|}
-91.03 \\
\end{tabular} & 2.25 & 0.2 & 2005 \\
\hline 149 & 71799 & -2.04 & -36.76 & 5.54 & 3.5 & 2012 \\
\hline 150 & 99294 & -2.03 & -88.19 & 2.3 & 0.27 & 2007 \\
\hline 151 & 71492 & -1.98 & -91.41 & 2.16 & 0.19 & 2003 \\
\hline 152 & 99248 & -1.96 & -95.45 & 2.06 & 0.09 & 2005 \\
\hline 153 & 71374 & -1.94 & -46.84 & 4.13 & 2.2 & 2005 \\
\hline 154 & 101769 & -1.94 & -30.68 & 6.31 & 4.38 & 2003 \\
\hline 155 & 99383 & -1.93 & -29.15 & 6.61 & 4.68 & 2011 \\
\hline 156 & 102744 & -1.93 & -76.4 & 2.52 & 0.59 & 2009 \\
\hline 157 & 99988 & -1.92 & -65.43 & 2.93 & 1.01 & 2005 \\
\hline 158 & 103028 & \begin{tabular}{|l|}
-1.91 \\
\end{tabular} & -99.79 & 1.91 & --- & 2005 \\
\hline 159 & 69670 & \begin{tabular}{|l|}
-1.89 \\
\end{tabular} & \begin{tabular}{|l|}
-98.8 \\
\end{tabular} & 1.91 & 0.02 & 2009 \\
\hline 160 & 99415 & \begin{tabular}{|l|}
-1.89 \\
\end{tabular} & \begin{tabular}{|l|}
-38.7 \\
\end{tabular} & 4.88 & 2.99 & 2009 \\
\hline 161 & 100540 & \begin{tabular}{|l|} 
\\
\end{tabular} & -93.22 & 2.01 & 0.14 & 2005 \\
\hline 162 & 102590 & -1.85 & -29.61 & 6.23 & 4.39 & 2009 \\
\hline 163 & 99872 & -1.83 & -86.58 & 2.11 & 0.28 & 2005 \\
\hline 164 & 102135 & -1.83 & -93.95 & 1.94 & 0.12 & 2009 \\
\hline 165 & 101394 & -1.82 & -48.51 & 3.76 & 1.93 & 2010 \\
\hline 166 & 101145 & -1.8 & -99.01 & 1.82 & 0.02 & 2003 \\
\hline \begin{tabular}{|l|}
167 \\
\end{tabular} & 99385 & \begin{tabular}{|l|}
-1.79 \\
\end{tabular} & -95.89 & 1.86 & 0.08 & 2002 \\
\hline \begin{tabular}{|l|}
168 \\
\end{tabular} & 102319 & \begin{tabular}{|l|}
-1.78 \\
\end{tabular} & -35.86 & 4.97 & 3.19 & 2007 \\
\hline 169 & 101628 & \begin{tabular}{|l|}
-1.78 \\
\end{tabular} & -50.53 & 3.52 & 1.74 & 2009 \\
\hline \begin{tabular}{|l|}
170 \\
\end{tabular} & 71602 & -1.75 & -31.76 & 5.52 & 3.77 & --- \\
\hline 171 & 99895 & -1.72 & -68.34 & 2.52 & 0.8 & 2005 \\
\hline 172 & 101675 & -1.7 & -98.62 & 1.73 & 0.02 & 2002 \\
\hline 173 & 102389 & -1.69 & -91.46 & 1.84 & 0.16 & 2012 \\
\hline
\end{tabular}




\begin{tabular}{|c|c|c|c|c|c|c|}
\hline 174 & 102548 & \begin{tabular}{|l|}
-1.69 \\
\end{tabular} & -94.39 & 1.79 & 0.1 & 2005 \\
\hline 175 & 101444 & -1.68 & -45.93 & 3.65 & 1.97 & 2007 \\
\hline 176 & 72794 & -1.67 & -72.55 & 2.3 & 0.63 & 2006 \\
\hline 177 & 70815 & -1.64 & -99.13 & 1.65 & 0.01 & 2005 \\
\hline \begin{tabular}{|l|}
178 \\
\end{tabular} & 71104 & \begin{tabular}{|l|}
-1.59 \\
\end{tabular} & -36.52 & 4.36 & 2.77 & 2013 \\
\hline 179 & 99198 & -1.56 & -30.79 & 5.06 & 3.5 & 2009 \\
\hline 180 & 102613 & -1.54 & -98.65 & 1.56 & 0.02 & 2004 \\
\hline 181 & 100010 & -1.52 & -98.08 & 1.55 & 0.03 & 2002 \\
\hline 182 & 73099 & -1.52 & -97.14 & 1.56 & 0.04 & 2012 \\
\hline 183 & 102372 & -1.49 & -97.81 & 1.52 & 0.03 & 2003 \\
\hline 184 & 102694 & -1.43 & -99.65 & 1.43 & --- & 2005 \\
\hline 185 & 102134 & -1.42 & -74.54 & 1.91 & 0.49 & 2003 \\
\hline 186 & 99249 & -1.4 & -93.97 & 1.48 & 0.09 & 2005 \\
\hline 187 & 102376 & -1.4 & -47.21 & 2.96 & 1.56 & 2007 \\
\hline 188 & 100200 & -1.38 & -36.28 & 3.8 & 2.42 & 2005 \\
\hline \begin{tabular}{|l|}
189 \\
\end{tabular} & 100541 & -1.35 & -92.47 & 1.46 & 0.11 & 2005 \\
\hline 190 & 72678 & -1.34 & -37.31 & 3.59 & 2.25 & 2012 \\
\hline 191 & 100502 & -1.32 & -91.81 & 1.44 & 0.12 & 2005 \\
\hline 192 & 102468 & -1.31 & -96.25 & 1.36 & 0.05 & 2009 \\
\hline 193 & 73410 & -1.28 & -99.44 & 1.29 & 0.01 & 2007 \\
\hline 194 & 101400 & -1.27 & -88.08 & 1.44 & 0.17 & -- \\
\hline 195 & 102471 & -1.27 & -70.03 & 1.81 & 0.54 & 2003 \\
\hline 196 & 100020 & -1.25 & -95.4 & 1.31 & 0.06 & 2002 \\
\hline 197 & 71292 & -1.24 & -68.3 & 1.81 & 0.57 & 2012 \\
\hline 198 & 101635 & -1.24 & -96.81 & 1.28 & 0.04 & 2005 \\
\hline 199 & 101397 & -1.23 & -59.96 & 2.05 & 0.82 & 2008 \\
\hline 200 & 102704 & -1.22 & -32.19 & 3.8 & 2.58 & 2006 \\
\hline 201 & 99863 & -1.22 & -75.24 & 1.62 & 0.4 & \begin{tabular}{|l}
-- \\
\end{tabular} \\
\hline 202 & 102002 & \begin{tabular}{|l|}
-1.19 \\
\end{tabular} & -59.86 & 1.98 & 0.8 & 2005 \\
\hline 203 & 101101 & -1.19 & -33.97 & 3.49 & 2.31 & 2005 \\
\hline 204 & 101289 & -1.19 & -96.15 & 1.24 & 0.05 & 2002 \\
\hline 205 & 100826 & -1.18 & -33.08 & 3.56 & 2.38 & 2012 \\
\hline 206 & 102609 & \begin{tabular}{|l|} 
\\
\end{tabular} & -93.77 & 1.25 & 0.08 & 2004 \\
\hline 207 & 99852 & -1.15 & -49.23 & 2.34 & 1.19 & 2005 \\
\hline 208 & 72079 & -1.15 & -56.77 & 2.03 & 0.88 & 2013 \\
\hline 209 & 101292 & -1.14 & -34.15 & 3.33 & 2.19 & 2012 \\
\hline 210 & 99276 & -1.14 & -93.83 & 1.21 & 0.07 & 2005 \\
\hline 211 & 99941 & -1.13 & -66.02 & 1.72 & 0.58 & 2007 \\
\hline 212 & 102192 & -1.12 & -33.85 & 3.3 & 2.18 & 2011 \\
\hline 213 & 103151 & -1.11 & -86.65 & 1.28 & 0.17 & 2010 \\
\hline 214 & 101701 & -1.11 & -30.89 & 3.6 & 2.49 & 2006 \\
\hline 215 & 99886 & -1.1 & -89.27 & 1.24 & 0.13 & 2005 \\
\hline 216 & 100806 & -1.09 & \begin{tabular}{|l|}
-92.9 \\
\end{tabular} & 1.18 & 0.08 & 2009 \\
\hline 217 & 102347 & -1.07 & -33.18 & 3.22 & 2.15 & 2009 \\
\hline 218 & 101829 & -1.06 & -27.03 & 3.91 & 2.85 & 2009 \\
\hline 219 & 99207 & -1.05 & -27.11 & 3.88 & 2.83 & 2002 \\
\hline 220 & 71535 & -1.05 & -95.97 & 1.1 & 0.04 & 2005 \\
\hline
\end{tabular}




\begin{tabular}{|c|c|c|c|c|c|c|}
\hline 221 & 101322 & -1.04 & -62.17 & 1.67 & 0.63 & 2002 \\
\hline 222 & 71732 & -1.02 & $\mid-51.24$ & 1.99 & 0.97 & 2013 \\
\hline 223 & 99371 & -1.01 & -60.32 & 1.68 & 0.67 & 2012 \\
\hline 224 & 99332 & -1.01 & -54.86 & 1.83 & 0.83 & 2005 \\
\hline 225 & 71606 & -0.99 & -58.59 & 1.68 & 0.7 & 2009 \\
\hline 226 & 100229 & \begin{tabular}{|l|}
-0.99 \\
\end{tabular} & -35 & 2.82 & 1.83 & --- \\
\hline 227 & 99268 & -0.98 & -36.25 & 2.71 & 1.73 & 2005 \\
\hline 228 & 99306 & \begin{tabular}{|l|}
-0.97 \\
\end{tabular} & \begin{tabular}{|l|}
-93.03 \\
\end{tabular} & 1.04 & 0.07 & 2005 \\
\hline 229 & 71094 & $\mid-0.96$ & -67.5 & 1.43 & 0.46 & --- \\
\hline 230 & 102014 & -0.95 & -42.74 & 2.23 & 1.28 & 2002 \\
\hline 231 & 102553 & $\mid-0.95$ & $\mid-93.21$ & 1.02 & 0.07 & 2005 \\
\hline 232 & 101925 & \begin{tabular}{|l|}
-0.94 \\
\end{tabular} & -55.64 & 1.68 & 0.75 & 2009 \\
\hline 233 & 102085 & -0.93 & -44.75 & 2.07 & 1.14 & 2005 \\
\hline 234 & 101864 & -0.91 & -86.91 & 1.05 & 0.14 & 2007 \\
\hline 235 & 99669 & -0.91 & -27.06 & 3.38 & 2.46 & --- \\
\hline 236 & 99668 & \begin{tabular}{|l|}
-0.89 \\
\end{tabular} & -26.68 & 3.34 & 2.45 & --- \\
\hline 237 & 101890 & \begin{tabular}{|l|}
-0.89 \\
\end{tabular} & -27.89 & 3.2 & 2.31 & 2003 \\
\hline 238 & 102488 & -0.88 & -47.74 & 1.84 & 0.96 & 2003 \\
\hline 239 & 102401 & -0.86 & -61.39 & 1.41 & 0.54 & --- \\
\hline 240 & 102272 & -0.85 & -36.92 & 2.31 & 1.46 & --- \\
\hline 241 & 102459 & -0.84 & -78.31 & 1.07 & 0.23 & 2004 \\
\hline 242 & 73433 & -0.84 & -56.32 & 1.49 & 0.65 & 2007 \\
\hline 243 & 102572 & -0.84 & -68.53 & 1.22 & 0.38 & 2004 \\
\hline 244 & 72867 & -0.82 & -69.15 & 1.19 & 0.37 & 2012 \\
\hline 245 & 99697 & -0.82 & -50.81 & 1.62 & 0.8 & 2007 \\
\hline 246 & 65855 & \begin{tabular}{|l|}
-0.79 \\
\end{tabular} & -36.69 & 2.16 & \begin{tabular}{|l|}
1.37 \\
\end{tabular} & 2004 \\
\hline 247 & 101777 & \begin{tabular}{|c|}
-0.77 \\
\end{tabular} & -51.69 & 1.48 & 0.72 & 2008 \\
\hline 248 & 102292 & -0.72 & -59.27 & 1.21 & 0.49 & 2009 \\
\hline 249 & 100078 & \begin{tabular}{|c|}
-0.68 \\
\end{tabular} & -32.73 & 2.08 & 1.4 & --- \\
\hline 250 & 102460 & -0.68 & -43.58 & 1.57 & 0.88 & --- \\
\hline 251 & 101107 & -0.66 & -40.41 & 1.64 & 0.98 & 2007 \\
\hline 252 & 99870 & -0.65 & -55.29 & 1.17 & 0.52 & 2004 \\
\hline 253 & 102749 & \begin{tabular}{|c|}
-0.64 \\
\end{tabular} & -53.49 & 1.2 & 0.56 & 2012 \\
\hline 254 & 101528 & \begin{tabular}{|l|}
-0.63 \\
\end{tabular} & -27.31 & 2.3 & 1.68 & 2001 \\
\hline 255 & 72279 & -0.6 & -30.57 & 1.96 & 1.36 & 2007 \\
\hline 256 & 101775 & \begin{tabular}{|c|}
-0.59 \\
\end{tabular} & -39.21 & 1.51 & 0.92 & 2011 \\
\hline 257 & 69671 & \begin{tabular}{|l|}
-0.59 \\
\end{tabular} & -35.78 & 1.66 & 1.07 & --- \\
\hline 258 & 101513 & -0.55 & -36.93 & 1.49 & 0.94 & --- \\
\hline 259 & 99643 & -0.52 & -43.77 & 1.19 & 0.67 & --- \\
\hline 260 & 69207 & -0.52 & -28.2 & 1.85 & 1.33 & 2005 \\
\hline 261 & 102157 & \begin{tabular}{|l|}
-0.48 \\
\end{tabular} & -27.72 & 1.75 & 1.26 & --- \\
\hline 262 & 99311 & -0.47 & -44.75 & 1.05 & 0.58 & 2010 \\
\hline 263 & 101759 & -0.46 & -27.98 & 1.64 & 1.18 & 2012 \\
\hline 264 & 100322 & -0.46 & -36.04 & 1.29 & 0.82 & 2014 \\
\hline 265 & 100910 & \begin{tabular}{|c|} 
\\
\end{tabular} & -27.79 & 1.54 & 1.11 & --- \\
\hline 266 & 72940 & -0.4 & -25.3 & 1.58 & 1.18 & 2010 \\
\hline 267 & 101630 & -0.32 & -25.38 & 1.28 & 0.95 & --- \\
\hline
\end{tabular}


Table S3: Annually aggregated observations of mean air temperature, accumulated precipitation, accumulated snowfall, accumulated freezing degree days, and freezing days per winter year (1 July through 30 June) for climate station Kotzebue, sorted by year. *Mean Air Temperatures for winter year 2019 are interpolated due to missing temperature measurements from 1 May through 3 September 2019.

\begin{tabular}{|c|c|c|c|c|c|c|c|c|}
\hline $\begin{array}{l}\text { Winter } \\
\text { Year }\end{array}$ & $\begin{array}{c}\text { Mean Air } \\
\text { Temperature } \\
{\left[{ }^{\circ} \mathrm{C}\right]}\end{array}$ & $\begin{array}{c}\text { Cumulative } \\
\text { Precipitation } \\
\text { [mm] }\end{array}$ & $\begin{array}{c}\text { Cumulative } \\
\text { Snowfall } \\
{[\mathrm{cm}]}\end{array}$ & $\begin{array}{c}\text { Cumulative } \\
\text { FDD }\end{array}$ & $\begin{array}{c}\text { Freezing } \\
\text { Days }\end{array}$ & $\begin{array}{c}\text { Rank } \\
\text { Temperature }\end{array}$ & $\begin{array}{c}\text { Rank } \\
\text { Precipitation }\end{array}$ & $\begin{array}{c}\text { Rank } \\
\text { Snowfall }\end{array}$ \\
\hline *2019 & -0.21 & 278.6 & 155.1 & --- & --- & 1 & 21 & 28 \\
\hline 2018 & -1.33 & 424.5 & 274.2 & -1904.8 & 196 & 2 & 1 & 2 \\
\hline 2017 & -3.01 & 225.0 & 136.7 & -2631.1 & 194 & 7 & 43 & 36 \\
\hline 2016 & -1.84 & 258.1 & 151.6 & -2142.9 & 200 & 3 & 31 & 30 \\
\hline 2015 & -2.34 & 247.8 & 63.6 & -2428.8 & 208 & 5 & 36 & 68 \\
\hline 2014 & -2.27 & 260.5 & 82.8 & -2136.8 & 178 & 4 & 30 & 62 \\
\hline 2013 & -5.41 & 402.0 & 95.3 & -3207.3 & 223 & 38 & 2 & 57 \\
\hline 2012 & -6.93 & 295.8 & 219.1 & -3662.4 & 222 & 61 & 17 & 8 \\
\hline 2011 & -3.76 & 387.4 & 263.9 & -2772.6 & 216 & 11 & 6 & 4 \\
\hline 2010 & -5.03 & 226.5 & 108.4 & -3098.2 & 223 & 29 & 42 & 52 \\
\hline 2009 & -5.93 & 390.0 & 271.9 & -3341.0 & 220 & 45 & 5 & 3 \\
\hline 2008 & -4.66 & 221.0 & 221.4 & -3109.2 & 218 & 20 & 44 & 7 \\
\hline 2007 & -4.83 & 272.7 & 168.8 & -2946.2 & 213 & 26 & 23 & 20 \\
\hline 2006 & -5.98 & 263.3 & 158.7 & -3468.6 & 216 & 47 & 27 & 24 \\
\hline 2005 & -4.00 & 268.3 & 304.7 & -2873.6 & 213 & 15 & 26 & 1 \\
\hline 2004 & -3.64 & 313.5 & 229.7 & -2698.2 & 181 & 10 & 13 & 6 \\
\hline 2003 & -2.73 & 244.0 & 172.6 & -2262.9 & 195 & 6 & 37 & 16 \\
\hline 2002 & -5.27 & 269.0 & 187.7 & -3174.1 & 217 & 32 & 25 & 10 \\
\hline 2001 & -4.31 & 327.6 & 175.2 & -2442.7 & 245 & 17 & 9 & 12 \\
\hline 2000 & -6.23 & 205.4 & 85.9 & -3426.1 & 229 & 52 & 59 & 61 \\
\hline 1999 & -6.22 & 324.3 & 188.6 & -3415.2 & 224 & 51 & 10 & 9 \\
\hline 1998 & -3.94 & 390.4 & 126.9 & -2809.2 & 208 & 14 & 4 & 38 \\
\hline 1997 & -5.35 & 252.7 & 147.0 & -3110.3 & 216 & 34 & 33 & 32 \\
\hline 1996 & -4.80 & 214.5 & 158.4 & -3090.8 & 209 & 24 & 46 & 25 \\
\hline 1995 & -5.92 & 393.7 & 113.4 & -3334.5 & 210 & 44 & 3 & 47 \\
\hline 1994 & -4.74 & 336.6 & 173.2 & -2878.6 & 208 & 23 & 8 & 15 \\
\hline 1993 & -5.74 & 243.8 & 148.1 & -3259.1 & 237 & 43 & 38 & 31 \\
\hline 1992 & -6.24 & 208.6 & 112.2 & -3477.4 & 222 & 53 & 53 & 48 \\
\hline 1991 & -5.44 & 377.8 & 230.3 & -3376.2 & 210 & 40 & 7 & 5 \\
\hline 1990 & -5.94 & 323.5 & 126.6 & -3552.6 & 207 & 46 & 11 & 39 \\
\hline 1989 & -5.37 & 277.9 & 184.5 & -3078.5 & 224 & 36 & 22 & 11 \\
\hline 1988 & -4.56 & 211.1 & 121.4 & -2871.3 & 198 & 19 & 50 & 43 \\
\hline 1987 & -4.96 & 271.2 & 103.5 & -2968.0 & 226 & 27 & 24 & 55 \\
\hline 1986 & -5.28 & 238.4 & 95.2 & -3060.9 & 227 & 33 & 39 & 58 \\
\hline 1985 & -6.10 & 284.3 & 124.2 & -3206.1 & 219 & 50 & 19 & 41 \\
\hline 1984 & -6.77 & 284.3 & 115.0 & -3443.6 & 236 & 59 & 20 & 46 \\
\hline 1983 & -4.06 & 231.5 & 98.5 & -2756.5 & 211 & 16 & 41 & 56 \\
\hline 1982 & -5.42 & 262.5 & 106.0 & -3098.9 & 233 & 39 & 29 & 53 \\
\hline 1981 & -3.84 & 169.5 & 105.4 & -2602.5 & 211 & 12 & 66 & 54 \\
\hline
\end{tabular}




\begin{tabular}{|c|c|c|c|c|c|c|c|c|}
\hline 1980 & -3.91 & 290.1 & 111.2 & -2818.7 & 196 & 13 & 18 & 49 \\
\hline 1979 & -3.29 & 207.5 & 64.3 & -2648.8 & 221 & 8 & 55 & 67 \\
\hline 1978 & -3.52 & 210.9 & 40.7 & -2795.1 & 206 & 9 & 51 & 70 \\
\hline 1977 & -5.47 & 157.8 & 128.4 & -3173.8 & 216 & 41 & 68 & 37 \\
\hline 1976 & -8.25 & 199.7 & 124.5 & -3923.1 & 239 & 69 & 62 & 40 \\
\hline 1975 & -7.17 & 214.0 & 109.3 & -3768.4 & 236 & 65 & 48 & 51 \\
\hline 1974 & -7.05 & 256.3 & 122.8 & -3497.1 & 238 & 63 & 32 & 42 \\
\hline 1973 & -5.35 & 209.2 & 170.3 & -3209.6 & 214 & 35 & 52 & 18 \\
\hline 1972 & -6.97 & 207.2 & 174.4 & -3658.1 & 227 & 62 & 56 & 14 \\
\hline 1971 & -7.96 & 160.3 & 109.6 & -3975.5 & 237 & 68 & 67 & 50 \\
\hline 1970 & -5.39 & 104.9 & 75.6 & -3074.4 & 205 & 37 & 70 & 65 \\
\hline 1969 & -4.50 & 146.9 & 88.2 & -3064.8 & 217 & 18 & 69 & 59 \\
\hline 1968 & -6.49 & 201.8 & 155.9 & -3414.4 & 223 & 56 & 61 & 27 \\
\hline 1967 & -4.68 & 179.6 & 164.7 & -2886.5 & 219 & 22 & 65 & 22 \\
\hline 1966 & -7.48 & 262.7 & 169.0 & -3642.3 & 240 & 66 & 28 & 19 \\
\hline 1965 & -6.55 & 193.0 & 174.7 & -3507.8 & 210 & 57 & 64 & 13 \\
\hline 1964 & -8.76 & 300.6 & 154.0 & -4130.0 & 227 & 70 & 15 & 29 \\
\hline 1963 & -5.07 & 248.2 & 146.9 & -3043.6 & 217 & 30 & 35 & 33 \\
\hline 1962 & -5.99 & 202.5 & 166.3 & -3273.7 & 222 & 48 & 60 & 21 \\
\hline 1961 & -5.53 & 300.4 & 137.7 & -3103.4 & 221 & 42 & 16 & 35 \\
\hline 1960 & -6.68 & 213.1 & 74.7 & -3420.9 & 220 & 58 & 49 & 66 \\
\hline 1959 & -6.27 & 237.4 & 81.3 & -3461.0 & 220 & 54 & 40 & 63 \\
\hline 1958 & -4.80 & 207.0 & 142.6 & -3037.2 & 215 & 25 & 57 & 34 \\
\hline 1957 & -6.44 & 196.0 & 163.3 & -3520.9 & 224 & 55 & 63 & 23 \\
\hline 1956 & -7.11 & 216.8 & 158.0 & -3712.5 & 212 & 64 & 45 & 26 \\
\hline 1955 & -7.48 & 305.9 & 120.4 & -3711.7 & 225 & 67 & 14 & 45 \\
\hline 1954 & -5.99 & 206.9 & 120.7 & -3285.1 & 214 & 49 & 58 & 44 \\
\hline 1953 & -5.17 & 214.3 & 62.6 & -2982.6 & 213 & 31 & 47 & 69 \\
\hline 1952 & -6.85 & 322.7 & 171.6 & -3485.5 & 236 & 60 & 12 & 17 \\
\hline 1951 & -5.01 & 250.8 & 87.8 & -3263.0 & 199 & 28 & 34 & 60 \\
\hline
\end{tabular}

\section{References}

GISTEMP Team, 2019: GISS Surface Temperature Analysis (GISTEMP). NASA Goddard Institute for Space Studies. Dataset accessed 2020-04-13 at data.giss.nasa.gov/gistemp/. 